\title{
Proyectos de indagación: su impacto en la competencia científica en estudiantes de Uruguay
}

Recibido: 10 de febrero de 2019 / Revisado: 12 de marzo de 2019

Aceptado: 29 de marzo de 2019 / Publicado: 22 de julio de 2019

DAISY IMBERT ROMERO,

Universidad Internacional Iberoamericana

daisyimbertromero@gmail.com

EDUARDO ELOSEGUI BANDERA

Universidad de Málaga

http://dx.doi.org/10.24310/IJNE2.1.2019.6561

\section{RESUMEN}

Se elaboraron secuencias didácticas a través de Proyectos de Indagación desde un enfoque dual, ya que se integraron aspectos epistemológicos y didácticos, con el objetivo de analizar la influencia de las mismas en el desarrollo de la competencia científica y aunar criterios cuando se diseñan dentro del modelo de aprendizaje por indagación.

La investigación acción se realizó en un liceo de contexto sociocultural desfavorable. Participaron tres docentes, con dos grupos a cargo cada una. Las docentes seleccionaron el mejor grupo y el de mayores dificultades al inicio del año lectivo, de los grupos que les fueron asignados por la institución, a los efectos de cubrir la mayor diversidad posible. Intervinieron 115 estudiantes.

La selección de docentes se efectuó por muestreo casual por pertenecer al centro en el año 2017. Dentro del grupo de docentes del liceo, la muestra se concretó en base

\section{ABSTRACT}

Inquiry-based projects: its impact on the scientific competence of students in Uruguay

Didactic sequences were elaborated through Inquiry Projects from a dual approach, since epistemological and didactic aspects were integrated, with the aim of analyzing the influence of these in the development of scientific competence and to combine criteria when they are designed within the model of learning by inquiry.

The action research was carried out in a lyceum with an unfavorable sociocultural context. Three teachers participated, with two groups in charge each. The teachers selected the best group and the one with the greatest difficulties at the beginning of the school year, from the groups that were assigned by the institution, in order to cover as much diversity as possible. 115 students participated. 
a otros criterios: docentes efectivas, con continuidad en dicho año lectivo, que tuvieran al menos dos grupos del mismo grado y asumieran este desafío. Se triangularon dos técnicas: análisis de documentos de las rúbricas aplicadas a la propuesta diagnóstica y a los pósteres, así como observación de clases y la observación durante la defensa de los pósteres.

Se pudo apreciar un avance en el desarrollo de las dimensiones de la competencia científica en los resultados logrados por los estudiantes de 4to año. Surge como dato inédito que se desvanecieron las diferencias iniciales detectadas por la propuesta diagnóstica en grupos del mismo grado.

Palabras Clave: Secuencias didácticas, competencia científica, proyectos de indagación, póster.
The selection of teachers was made by casual sampling to belong to the center in the year 2017. Within the group of teachers of the lyceum, the sample was made based on other criteria: effective teachers, with continuity in that school year, who had at least two groups of the same grade and take up this challenge. Two techniques were triangulated: analysis of documents of the rubrics applied to the diagnostic proposal and to the posters, as well as observation of classes and observation during the defense of the posters.

An advance was seen in the development of the dimensions of scientific competence in the results achieved by the students 4 th year, it arises as unpublished data that the initial differences detected by the diagnostic proposal in groups of the same degree vanished.

Keywords: Didactic sequences, scientific competence, inquiry-based projects, post.

\section{Introducción}

Actualmente constituye un desafío planificar los contenidos curriculares para que sean atractivos a estudiantes de este siglo caracterizado por el exceso de información, el cambio permanente y la incertidumbre.

Cañal (2011) refiere a que se debe superar la forma de enseñanza vigente, que el énfasis debe estar en la movilización de los conocimientos, pasar de estudiantes pasivos que solamente escuchan a discentes activos y competentes. Los educandos deben hallarse en un aula que propicie la formulación de preguntas y la toma de decisiones sobre qué investigar.

Este cambio en las formas de aprender a través de la producción de preguntas para investigar, por parte de los estudiantes, implican transformaciones en la forma de enseñar. Los docentes tienen que facilitar ese espacio, para enseñar a través de las investigaciones que realizan los discentes. Para ello las estrategias de investigación deberán incorporarse como estrategias didácticas para que los educandos puedan desarrollar la competencia científica.

Tobón, Pimienta y García (2010), Zabala y Arnau (2014), Cañal (2011), Quintanilla (2014) y Pedrinaci (2012), entre otros autores, si bien manifiestan algunas diferencias en sus planteos, todos ellos 
coinciden en ciertos aspectos que deben cambiar en las instituciones educativas, en relación al abordaje del currículo, para el logro de estudiantes competentes. Ellos son, una enseñanza de la naturaleza de las ciencias en las que estén implicadas, la resolución de problemas, la contextualización, la toma de decisiones por parte de los estudiantes y el avanzar hacia el aprender a aprender.

El presente artículo que forma parte de la tesis doctoral, comunica una investigación en didáctica en la que se diseñaron proyectos de indagación o de introducción a la investigación (PII), a partir de los contenidos curriculares, con la finalidad de lograr el avance en las distintas dimensiones de la competencia científica.

En un estudio efectuado por Chin y Osborne (2008) obtuvieron como resultado que un $75 \%$ de los educandos de 6to año eligieron investigar preguntas elaboradas por ellos mismos. Describieron el trabajo como "emocionante, divertido e interesante" lo que indica la importancia de partir de la curiosidad de los estudiantes, así como plantearse preguntas y desarrollar la habilidad de reconocer cuales son probables de ser investigadas.

Los mismos resultados se obtuvieron en una investigación realizada en España, en la que surge que los implicados con este modelo estuvieron más motivados, como expresan los docentes: "los niños, incluso los repetidores o de notas bajas, los ves animados y les gusta bastante" (Travé, Pozuelo, Cañal, 2007, p. 16)

\subsection{Competencia científica}

Uno de los propósitos a los que se propende corresponde a que los estudiantes durante el transcurso del proyecto puedan avanzar en el desarrollo de las diferentes capacidades y dimensiones de la competencia científica.

Pedrinaci (2012, p. 31) la define de la siguiente manera:

Conjunto integrado de capacidades para utilizar el conocimiento científico a fin de describir, explicar y predecir fenómenos naturales; para comprender los rasgos característicos de la ciencia; para formular e investigar problemas e hipótesis; así como para documentar, argumentar y tomar decisiones personales y sociales sobre el mundo natural y los cambios que la actividad humana genera en él.

La definición integra: conocimiento de la ciencia, práctica de la ciencia y naturaleza de la ciencia vinculada con la tecnología y la sociedad. 
En esta investigación se toman en cuenta además las capacidades y dimensiones de la competencia científica elaboradas por Franco Mariscal (2015).

\subsection{Proyectos de Indagación}

Cuando se menciona el trabajo con Proyectos, los mismos implican diversos tipos. Puede tratarse de Proyectos de producción, tecnológicos, sociales, o de investigación entre tantos otros, en el presente artículo se hace referencia a los últimos. Por tratarse de investigación escolar se los denomina como Proyectos de Introducción a la Investigación (PII) o Proyectos de Indagación.

Las cualidades del trabajo con Proyectos pueden apreciarse en las investigaciones a las que se refieren los antecedentes revisados.

Sánchez (2013) indica que para los discentes la experiencia les posibilitó desarrollar su proceso de aprendizaje. Tener la posibilidad de seleccionar las actividades a partir de su interés, favoreció la profundización en los contenidos del curso, así como sentirse responsables por el éxito de la actividad, facilitó el aprendizaje de todos los estudiantes y la integración de los mismos y permitió el aprendizaje interdisciplinar. Los proyectos cumplieron con exigencias de alta calidad.

Caamaño y Corominas (2004) en tanto se refieren a que una forma de conectar "la ciencia escolar" y "la ciencia de la calle" es partir del contexto, denominado enfoque basado en el contexto, que está siendo muy utilizado en proyectos británicos. Considera importante preguntar “¿qué problemas se van a abordar con los estudiantes como ciudadanos o como futuros científicos?" Asimismo, enfatizan la importancia de propuestas abiertas y como debe ser la estructura de los guiones escritos que se les brinda a los estudiantes a los efectos de alejarse de las "recetas de cocina" utilizadas frecuentemente.

Esto último es lo que se pretende lograr a través de los Proyectos de Introducción a la Investigación, que los estudiantes puedan identificar problemas en su contexto, plantearse preguntas y a través de los proyectos aprender los contenidos, conceptuales, procedimentales, epistémicos y actitudinales, un aprendizaje que les resulte útil fuera del liceo.

\subsection{Secuencias didácticas}

Couso (2013) afirma que últimamente existe un marcado interés por las secuencias didácticas, también denominadas secuencias de enseñanza y aprendizaje (SEA).

Una SEA es una planificación de un tema curricular, en la cual se introduce los recursos, las fichas de trabajo, los contenidos, el contexto, el orden de las actividades, y la evaluación de las mismas. 
Las secuencias además de ser muy importantes para el docente, son de interés para la investigación en didáctica y actualmente con el enfoque en el desarrollo de competencias, se ha reiniciado la discusión sobre el diseño de las mismas.

Couso (2011) indica que tradicionalmente el diseño ha contemplado dos enfoques: el epistemológico y el didáctico. La primera se centra en la ciencia y su relación con el mundo, la segunda pone el énfasis en el aprendiz y su vinculación con el mundo. Muchos autores han criticado esa dicotomía, por ello actualmente se está esgrimiendo un enfoque dual que conjuga ambos extremos.

Caamaño (2012) propone una secuencia en la que se observa un enfoque dual. El problema planteado se refiere a elegir el tejido que abriga más a partir de tres muestras correspondientes a algodón, lana y acrílico, de diferentes grosores. Se solicita al estudiante que piense el diseño metodológico, registre las medidas, elabore una conclusión y comunique la misma a través de un informe y de manera oral.

Por otra parte, Franco Mariscal, Blanco López y España Ramos (2014) planificaron una secuencia didáctica sobre salud bucal, que se organizó de la siguiente forma: La primera tarea parte de la interrogante “Por qué es importante cuidarse la boca?” La misma intenta explicitar las ideas previas. La consigna de la tarea consiste en justificar con cual persona se relacionaría a partir de la observación de fotografías. En la segunda tarea se plantea la pregunta “¿Cuáles son los factores que intervienen en la aparición de la caries?" Se espera que el estudiante constate dos factores: la ingesta de alimentos con azúcar y las bacterias en la boca. La tercera tarea parte de la cuestión “¿cómo se producen las caries?" se propone a los estudiantes que formulen hipótesis y posteriormente corroborar las mismas, a través de experiencias diseñadas por ellos. Arribaron a la conclusión que la presencia de azúcar como único factor no produce caries. Estos resultados y conclusiones fueron utilizados para discutir la validez de la conclusión y si se habían controlado todas las variables que pueden incidir. En la cuarta tarea parte de la pregunta "¿Qué hizo el dentista en tu última revisión?" Es útil para promover la reflexión sobre efectuar visitas periódicas al dentista y la interpretación de la radiografía dental mediante una teatralización. En la quinta tarea se plantea “¿Por qué debo cepillar mis dientes?" Previamente debe averiguar sobre los componentes principales de un dentífrico y sus funciones. Debe reconocer los ingredientes a partir de la etiqueta de varias marcas comerciales. En la sexta tarea la cuestión presentada es: “¿Qué ocurre si abuso de alimentos azucarados?” Para abordar el trabajo los estudiantes deben conocer la cantidad diaria de azúcar que consume cada uno de ellos. La séptima tarea plantea “¿Cómo puedo saber si el contenido de una página web es de calidad y fiable?” La pregunta en la octava tarea es: ¿Cómo influyen el tabaco, alcohol y otras drogas en la aparición de la caries? Se propende a que los estudiantes busquen información sobre la influencia de ciertas drogas en la formación de caries y que argumente a favor o no. En la novena tarea la interrogante corresponde a “Qué he aprendido sobre el problema de la caries?” el autor considera que la fase de evaluación también se implementa desde el enfoque de competencias, el trabajo consiste en la elaboración de un tríptico sobre el problema de las caries y la salud bucodental, donde el estudiante debe sintetizar lo aprendido. 
Zabala y Arnau (2014) plantean secuencias didácticas dentro del método de investigación en el medio. Una de ellas se inicia con la interrogante “¿cuánto papel gastamos?”. Las autoras indican que "La finalidad de toda la secuencia es el compromiso con el medio ambiente y con el reciclaje" (Zabala, Arnau, 2014, p. 109)

En esta breve revisión de antecedentes se pueden apreciar planteamientos que buscan el desarrollo de la competencia científica a través del diseño de secuencias didácticas. El desafío actual es lograr proyectar secuencias con un enfoque dual que posibiliten cambios y mejoras en el aprendizaje, que sean inclusivas, transformadoras y emancipadoras.

\subsection{Objetivos}

1. Desarrollar secuencias didácticas en consenso con los docentes, desde un enfoque dual a través del diseño de proyectos dentro del modelo didáctico de aprendizaje por indagación, a partir de temas curriculares y del interés de los estudiantes.

2. Describir el avance que logran realizar los estudiantes de los grupos seleccionados, en las distintas dimensiones y capacidades que incluye la competencia científica.

\section{Material y método}

Correspondió a una investigación-acción, enmarcada en la tesis de doctorado. Se realizó en el año 2017 desde febrero a diciembre, en un liceo de contexto sociocultural desfavorable. La tipificación del liceo surge de las categorizaciones que maneja el Consejo de Educación Secundaria (CES) en Uruguay.

Se organizó un equipo de trabajo colaborativo con tres docentes y se implementó la investigación en 6 grupos, dos de cada docente, un total de 115 estudiantes.

La selección de los docentes, se realizó por muestreo empírico, ya que las docentes involucradas trabajan en el liceo seleccionado. Otros criterios utilizados correspondieron a que fueran efectivas, tuvieran como mínimo dos grupos en un grado, su permanencia en el año lectivo y asumieran el desafío. De acuerdo a los criterios señalados se trabajó con tres grados (1ero, 3ero y 4to).

Respecto a los grupos se acordó, que las docentes seleccionaran de sus grupos (los grupos son adjudicados en elección de horas del CES) los que las mismas estimaron como el mejor grupo y el que tuviera mayores dificultades en cada grado, a los efectos de abarcar la mayor diversidad posible.

Se planteó una propuesta diagnóstica al inicio del año lectivo (marzo) para evaluar el nivel, en las diferentes dimensiones de la competencia científica, que presentaban los estudiantes al comenzar el 
curso. Posteriormente se comparó dicho nivel con el alcanzado al presentar los pósteres de los proyectos desarrollados (septiembre) y con otra prueba individual al finalizar el año lectivo.

Se trabajó durante el año en el diseño de secuencias a partir de los temas curriculares, correspondiendo cada secuencia a un Proyecto. Se observaron 18 clases en las que las docentes desarrollaron las secuencias. Se participó además en la observación de la Muestra de Proyectos donde comunicaron sus investigaciones a la comunidad. El ciclo de investigación-acción correspondió a tres secuencias-proyectos. Al finalizar cada ciclo se tomó la opinión de estudiantes y docentes a través de "focus group" a los efectos de mejorar la siguiente secuencia-proyecto.

En el presente artículo se hace referencia únicamente a la elaboración de la primera secuencia para los grupos de 4to año 1 y 2 , con un total de 44 estudiantes y se analiza el trabajo de una de las tres docentes. Se triangularon o "cristalizaron" (Richardson, 1997) los datos obtenidos en la propuesta diagnóstica inicial, con las clases observadas y los pósteres presentados en la Muestra.

\section{Resultados}

\subsection{Propuesta diagnóstica}

La propuesta diagnóstica constó de nueve ítems, los que permitieron evaluar las diferentes dimensiones de la competencia científica. En este artículo se hará referencia al primer ítem ya que el mismo se toma como punto de partida para la primera secuencia de 4to año, y al ítem que evalúa el diseño metodológico, que se comparará con lo logrado y evaluado a través de los posters.

\subsubsection{Identificar, explicar y utilizar fenómenos y evidencia científica.}

La situación problema planteada fue la siguiente: "Juan escuchó una conversación del padre con un pintor. El padre estaba solicitando que pintaran una habitación porque tenía hongos y el pintor explicaba al padre que los hongos provenían de la humedad que tenía la pared”.

De acuerdo a lo que has estudiado en el liceo indica: 1. La explicación del pintor tiene una base científica. Sí/ No. 2. Redacta una explicación para lo acontecido. 3. Indica que evidencia científica puedes utilizar.

Mediante esta situación problema se pretende evaluar las capacidades de: Identificar cuestiones científicas, explicar fenómenos científicamente y utilizar evidencia científica.

La evaluación del diagnóstico se efectúo empleando una rúbrica, que constó de nueve categorías. En la tabla 1 se observan los descriptores solo para la primera categoría. 
Tabla 1. Primera categoría de la rúbrica para evaluar primera actividad de propuesta diagnóstica. Fuente propia.

\begin{tabular}{|c|c|c|c|c|}
\hline $\begin{array}{c}\text { Categoría/ } \\
\text { Niveles }\end{array}$ & Excelente & Muy bueno & Aceptable & Debe Mejorar \\
\hline $\begin{array}{l}\text { Identificar } \\
\text { cuestiones } \\
\text { científicas } \\
\text { Explicar } \\
\text { fenómenos } \\
\text { científicamente } \\
\text { Utilizar eviden- } \\
\text { cia científica. }\end{array}$ & $\begin{array}{l}\text { Identifica que la conversa- } \\
\text { ción no tiene fundamento } \\
\text { científico ya que se refiere } \\
\text { a las condiciones de vida } \\
\text { de un ser vivo. Logra } \\
\text { explicar el origen de un } \\
\text { ser vivo a partir de otro ser } \\
\text { vivo. Refiere a la evidencia } \\
\text { científica de observar al } \\
\text { MO el moho. }\end{array}$ & $\begin{array}{l}\text { Identifica que la } \\
\text { conversación no tiene } \\
\text { fundamento científico } \\
\text { ya que se refiere a las } \\
\text { condiciones de vida } \\
\text { de un ser vivo. Logra } \\
\text { explicar el origen de } \\
\text { un ser vivo a partir de } \\
\text { otro ser vivo. No refiere } \\
\text { evidencia científica. }\end{array}$ & $\begin{array}{l}\text { Identifica que la } \\
\text { conversación no tiene } \\
\text { fundamento científico } \\
\text { ya que se refiere a las } \\
\text { condiciones de vida } \\
\text { de un ser vivo. No lo- } \\
\text { gra explicar ni utilizar } \\
\text { evidencia científica. }\end{array}$ & $\begin{array}{l}\text { No identifica que } \\
\text { la conversación no } \\
\text { tiene fundamento } \\
\text { científico, no logra } \\
\text { explicar ni utilizar } \\
\text { evidencia científica. }\end{array}$ \\
\hline
\end{tabular}

Tabla. 2. Resultados propuesta diagnóstica para el ítem identificar cuestiones científicas, explicar fenómenos científicamente y utilizar evidencia científica. Fuente propia.

\begin{tabular}{|c|c|c|c|c|}
\hline No estudiantes/ grupos & 4 to 1 & $\%$ & 4 to 2 & $\%$ \\
\hline Aceptable & 16 & 69,6 & 12 & 57,1 \\
\hline Muy bueno & 1 & 4,3 & 4 & 0 \\
\hline Excelente & 4 & 17,4 & 16 & 76,2 \\
\hline Subtotal suficientes & 21 & 91,3 & 5 & 23,8 \\
\hline Insuficientes & 2 & 8,7 & 21 & 100 \\
\hline Total estudiantes grupo & 23 & 100 & & 0 \\
\hline
\end{tabular}

En la tabla 2 pueden apreciarse los resultados obtenidos en la propuesta diagnóstica para esta categoría, en el grupo de 4to año 1, que la docente considera el mejor, la cantidad de estudiantes que lograron suficiente fue de 21 y el porcentaje $91,3 \%$, resultados superiores al grupo 4 to 2 .

En el grupo 1 veintiún estudiantes logran aceptable o más $(91,3 \%)$, en tanto en el grupo 2 son dieciséis estudiantes $(76,2 \%)$. Asimismo, en el grupo 1, cuatro estudiantes obtienen excelente $(17,4 \%)$, mientras que en el grupo 2 ningún estudiante logra el excelente.

\subsubsection{Diseño metodológico}

La propuesta diagnóstica evalúa ésta dimensión de la competencia científica junto a la capacidad de identificar variables, y realizar experiencias, a través del siguiente ítem: "De acuerdo a la pregunta investigable que planteaste, identifica las variables. Explica cómo procederás para responder la pregunta investigable”. Se solicitó en un ítem anterior que redactara una pregunta que pudiera responder con un trabajo de campo. 
Los resultados obtenidos en esta dimensión, luego de aplicada la rúbrica, se aprecian en la tabla 3.

Tabla 3. Resultados obtenidos por los estudiantes en la dimensión sobre diseño metodológico. Fuente propia.

\begin{tabular}{|c|c|c|c|c|}
\hline No estudiantes/grupos & 4 to 1 & $\%$ & 4 to 2 & $\%$ \\
\hline Aceptable & 13 & 56,5 & 5 & 23,8 \\
\hline Muy bueno & 4 & 17,4 & 6 & 28,6 \\
\hline Excelente & 4 & 17,4 & 6 & 81 \\
\hline Subtotal suficientes & 21 & 91,3 & 4 & 19 \\
\hline Insuficientes & 23 & 8,7 & 21 & 100 \\
\hline Total estudiantes grupo & 23 & 100 & 6 & 2 \\
\hline
\end{tabular}

El grupo 1 obtiene mejores resultados que el 2 igual que en el ítem analizado anteriormente, en tanto el 1 logra 91,3\% de resultados suficientes, el grupo 2 alcanza el 81\%. No obstante, si observamos los resultados excelentes son seis estudiantes que lo logran en el grupo $2(28,6 \%)$ y cuatro estudiantes en el grupo $1(17,4 \%)$

\subsection{Secuencia didáctica}

Cada una de las secuencias se organizó desde un enfoque dual que tuvo en cuenta tanto aspectos didácticos como epistemológicos. De acuerdo a ello se partió de problemáticas del contexto que permitieran abordar los contenidos curriculares, se priorizó el interés de los estudiantes en la elección de las variables involucradas, y se utilizaron tanto estrategias didácticas como estrategias propias de la metodología de investigación. En el proceso se logró la elaboración de cinco Proyectos de Indagación, uno para cada secuencia. En la tabla 4 se presenta la secuencia que parte de dicha pregunta. Se explicita la primera secuencia didáctica, con las diferentes actividades realizadas en cada clase, para lograr desarrollar el proyecto.

La secuencia se desarrolló en cinco clases, se abordaron los contenidos conceptuales de la unidad 1 y las diferentes dimensiones de la competencia científica en el desarrollo del proyecto. Los estudiantes al finalizar la secuencia elaboraron posters.

En una de las clases observadas, a partir de la situación problema sobre los hongos de la pared, se propone la pregunta investigable generadora ¿Qué condiciones ambientales se necesitan para que crezcan hongos en el trozo de pared recolectado o sobre otro sustrato?

Esta pregunta investigable generadora posibilita que la docente utilizando la interrogación didáctica vaya recabando diferentes condiciones que mencionan los estudiantes y realice un registro en la pizarra. 
Posteriormente la docente los invita a qué cada subgrupo seleccione la variable y el sustrato que desea investigar y que formulen sus preguntas investigables. Esta decisión que pueden tomar los estudiantes es muy importante, ya que, si bien el tema de investigación fue decidido por la docente, que los discentes puedan elegir las variables, implica tomar en cuenta sus intereses, lo que repercute en la motivación intrínseca (Alonso Tapia, 2005) y el aprendizaje. El uso de la app mentimeter y los celulares es un plus en la motivación.

Las preguntas investigables que elaboraron los estudiantes en la primera instancia fueron las siguientes: “¿En qué condiciones crecen los hongos con mayor rapidez en frutas y verduras?” (N, V y Y) “¿En qué condiciones un hongo descompone la fruta más rápido?” (F, M, B y S) “¿Cuál es el período de formación de hongos en las frutas seleccionadas?” (E, V y L) “¿Por qué surge el hongo en la fruta?” (E, N y H) “¿En qué condiciones surge la vida en la carne?” (M, C y A) “¿En cuánto tiempo surge la vida en la carne putrefacta?” (N, P y D) “¿En qué condiciones aparece el hongo en la fruta?” (S, F y G).

Puede apreciarse que muchas de las preguntas necesitan la orientación del docente para su reformulación ya que no cumplen con los requisitos para ser preguntas investigables (Arango, et al, 2009). Las que refieren a las condiciones deberán acotarse para trabajar una sola variable, ya que dentro del concepto de condiciones hay muchas involucradas y lo recomendable es que la pregunta se refiera a una sola variable independiente para facilitar la medición de la dependiente.

La pregunta “¿por qué surge el hongo en la fruta?” tampoco es investigable ya que conocer lasrazones por las cuales "surgen" los hongos, implica a muchos factores que pudieron incidir. De nuevo deberán acotar la pregunta a una variable dependiente e independiente.

En la pregunta “¿En qué condiciones surge la vida en la carne?” y en la anterior, se puede apreciar información sobre las ideas previas que puede tener el estudiante. No han superado la idea de la generación espontánea. Estos estudiantes con la orientación de la docente y el marco teórico trabajado, posteriormente reformularon la pregunta de esta forma ¿En qué tipo de carne aparecen primero las larvas? Experimentaron con carne de pollo, pescado y vaca, además utilizaron un frasco testigo que colocaron en la heladera. Si bien es importante que trabajen con testigos, también lo es que identifiquen las variables, en este caso no perciben que hay dos variables involucradas, además de ubicar el testigo en un lugar cerrado, variaron la temperatura, lo que puede determinar conclusiones no válidas.

En tanto la pregunta que se encuentra en la figura 1 “Cuál es el período de formación de hongos en las frutas seleccionadas?" involucra a una variable dependiente e independiente, por un lado, se medirá el tiempo y por otro lado se seleccionarán las frutas con las que se quiere investigar. 
Figura 1. Pregunta investigable inicial de estudiantes de 1ero de bachillerato (4to año). Fuente propia.

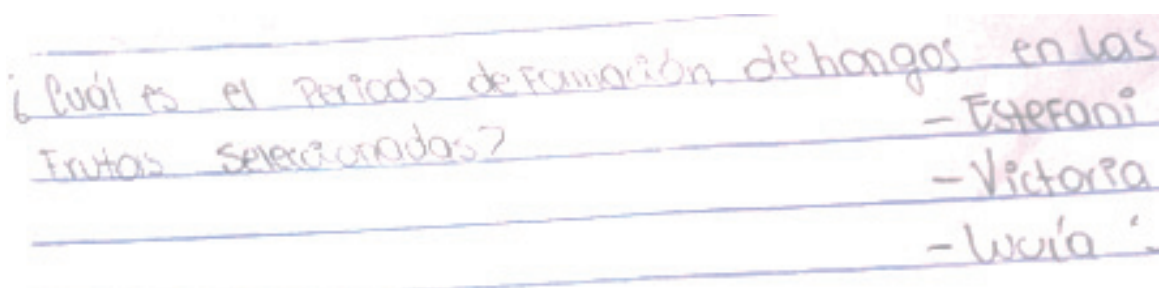

Se trata de una pregunta acotada e investigable. Deberán controlarse las demás variables que inciden en el proceso.

De las siete preguntas elaboradas en este grupo, solamente dos de ellas fueron investigables, las demás necesitaron la orientación de la docente para que se reformularan. Estos resultados indican la dificultad que presenta esta dimensión de la competencia científica.

\subsection{Comunicación de los Proyectos}

El 12 de septiembre de 2017 los estudiantes presentaron sus Proyectos de Introducción a la Investigación a la comunidad educativa del liceo, que además abrió sus puertas para recibir a escolares de diferentes escuelas y a la comunidad en general.

Se integró un jurado con las tres docentes y la investigadora. Se utilizaron escalas para la evaluación del póster y la defensa.

El evento de presentación de los trabajos atañe a una de las dimensiones de la competencia científica. Presentar los trabajos involucra ya sea la comunicación oral como la escrita.

Asimismo, a través de los posters se pueden evaluar otras dimensiones de la competencia científica como la redacción de las preguntas investigables, las hipótesis, la metodología, las conclusiones y si refiere correctamente la bibliografía.

En el póster se tomaron en cuenta las siguientes categorías para la evaluación: síntesis de las ideas centrales, organización de la información, competencia científica, originalidad y creatividad, ortografía y sintaxis.

Las categorías que se evaluaron en la defensa fueron: conocimiento sobre el tema, diseño metodológico y participación. 
Tabla 4. Primera secuencia didáctica para primero de bachillerato. Fuente propia.

\section{Pregunta investigable generadora}

¿Qué condiciones ambientales se necesitan para que crezcan hongos en el trozo de pared recolectado o sobre otro sustrato?

\section{Preguntas problemas}

¿Qué condiciones tuvo en cuenta Redi para refutar la teoría de la generación espontánea?

¿Qué otras variables deben tenerse en cuenta para realizar el experimento de Redi?

Competencias: todas las capacidades de la competencia científica. Competencia lingüística, competencia matemática y competencia digital.

Estrategias: problematización, ideas previas, contextualización, recurrencia y espiralización, trabajo colaborativo, interrogación didáctica, historia de las ciencias y estrategias de investigación.

Contenidos conceptuales: Se abordarán los contenidos de la unidad 1: Distintas teorías sobre el origen de la vida. Evolución prebiótica, hipótesis de Oparín. Moléculas precursoras de la vida.

\section{Actividad experimental CENTRAL:}

Experiencia de Redi o similares teniendo en cuenta las siguientes variables: temperatura, humedad, tipo de carne u otro substrato, luminosidad, tipo de recipiente, entre otras.

Temporalización: marzo y abril.

Evaluación formativa y formadora: actividades de clase, tareas domiciliarias, diferentes entregas del primer proyecto con sugerencias para mejorar, autoevaluación, evaluación mutua y entrega final del proyecto, elaboración de poster y ppt con defensa oral.

Instrumento de evaluación: Listas de cotejo, escalas y rúbrica.

\section{Clases - actividades}

1.Actividad 1. Se retoma situación problema del diagnóstico y se proyectan imágenes. Planteo de preguntas, identificación de variables.

Actividad 2. Formación de grupos, elección de tema para investigar, elaboración de la pregunta investigable para cada grupo e hipótesis. Entregar rúbrica.

Tarea domiciliaria: buscar información sobre experiencia de Redi en tres fuentes y realizar un marco teórico de una carilla. Subir a la plataforma.

2.Actividad 1. Puesta en común de los marcos teóricos, evaluación mutua de contenidos conceptuales y de capacidad de elaborar marco teórico utilizando la rúbrica.

Actividad 2. Pensar diseño metodológico para la pregunta de cada equipo (materiales y procedimiento)

Tarea domiciliaria: corregir marco teórico, buscar información sobre otras dos teorías de origen de la vida de dos fuentes y agregar al marco teórico, hasta 2 carillas. Subir a la plataforma.

3.Actividad 1. Implementación de la experiencia. Grabar videos.

Actividad 2. Actividad grupal: Texto para marcar las citas y las paráfrasis con colores distintos, sobre teorías de origen de la vida en una carilla. Elegir una teoría y explicársela al compañero. Puesta en común. Esquema en la pizarra de las teorías.

Tarea domiciliaria: Corregir marco teórico. Buscar información sobre otras dos teorías usando dos fuentes y agregar al marco teórico hasta 3 carillas. Realizar observaciones diarias de los experimentos, registrar con fotos y videos (cuando lo amerite) Subir a la plataforma.

4.Actividad 1. Observación de los experimentos, relevamiento con fotos o videos, registro de datos, elaboración de tablas de datos y gráficas.

Actividad 2. Evaluación mutua de marcos teóricos.

Actividad 3. Comenzar a redactar en grupos la discusión y conclusión.

Tarea domiciliaria: Continuar con observaciones y registro de datos. Elaborar informe en el que conste: pregunta de investigación, hipótesis, marco teórico, antecedentes, metodología, resultados, discusión, conclusión y referencias bibliográficas. Subir a la plataforma.

5.Actividad 1. Puesta en común de las discusiones y conclusiones: evaluación mutua.

Actividad 2. Comenzar a preparar póster.

Tarea domiciliaria: elaborar póster digital, y afiches para distribuir en la comunidad.

Exponer las investigaciones a otro grupo del liceo o a un grupo escolar. 
Cada categoría se evaluó con un valor máximo de 10 puntos. En el caso de los posters las cuatro integrantes del jurado debatieron hasta llegar a un puntaje para cada categoría ya que la evaluación se realizó sin la presencia de estudiantes. La evaluación de las defensas se efectúo diferente, por estar en presencia de los estudiantes. Por esa razón cada evaluadora adjudicó un puntaje y luego se promediaron los mismos.

\subsubsection{Posters}

Los pósteres elaborados por los estudiantes cumplieron con ciertas características de posters científicos: la información se dispuso en dos columnas, en el cuadrante superior izquierdo ubicaron el resumen y en el inferior derecho las conclusiones. Los resultados fueron presentados en tablas y gráficos, entre otros aspectos. Lo que puede apreciarse en la figura 2, 3 y 4 .

En la Muestra se presentaron los pósteres de todas las secuencias didácticas trabajadas, de la primera secuencia que es la que se analiza en este artículo, los pósteres representativos fueron: invasión de larvas y hongos en la fruta, los demás corresponden a las otras secuencias de primero bachillerato.

Figura 2. Póster elaborado por los estudiantes de 1ero de Bachillerato (4to año). Fuente propia.

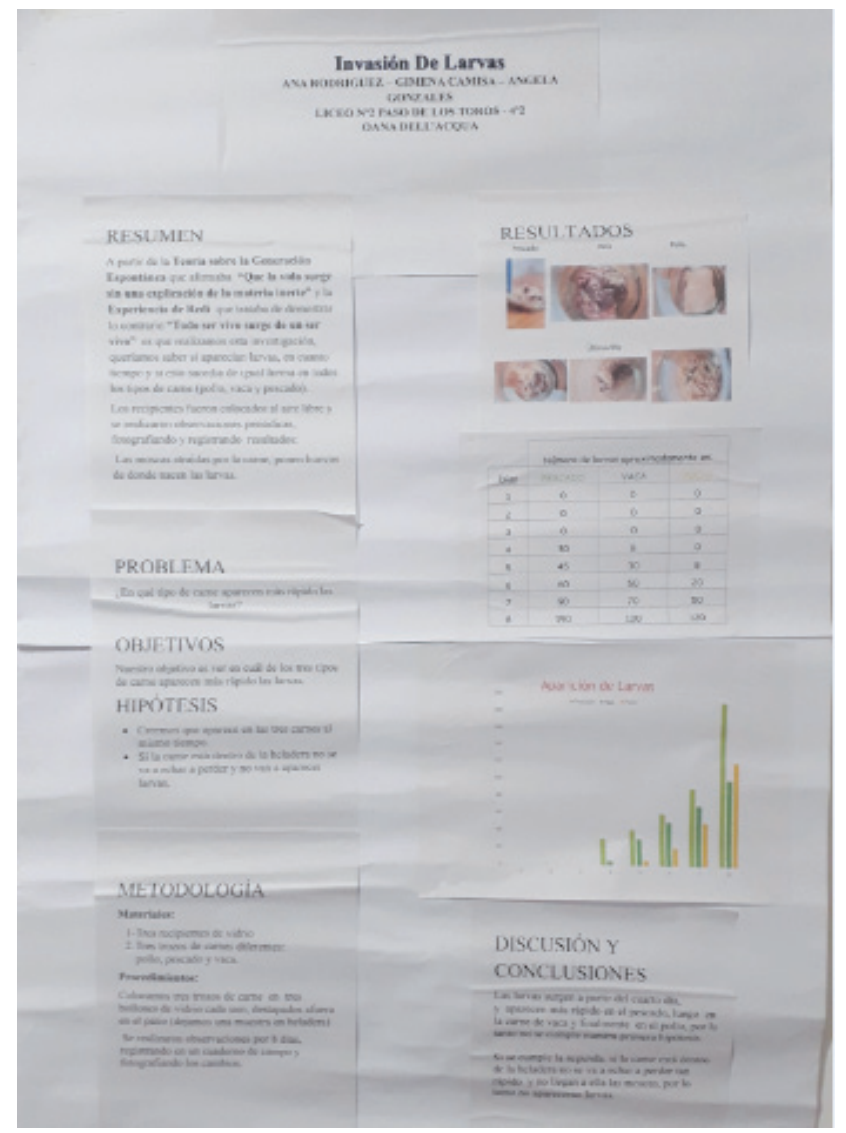

Figura 3. Presentación de datos en tablas y gráficos. Fuente propia.

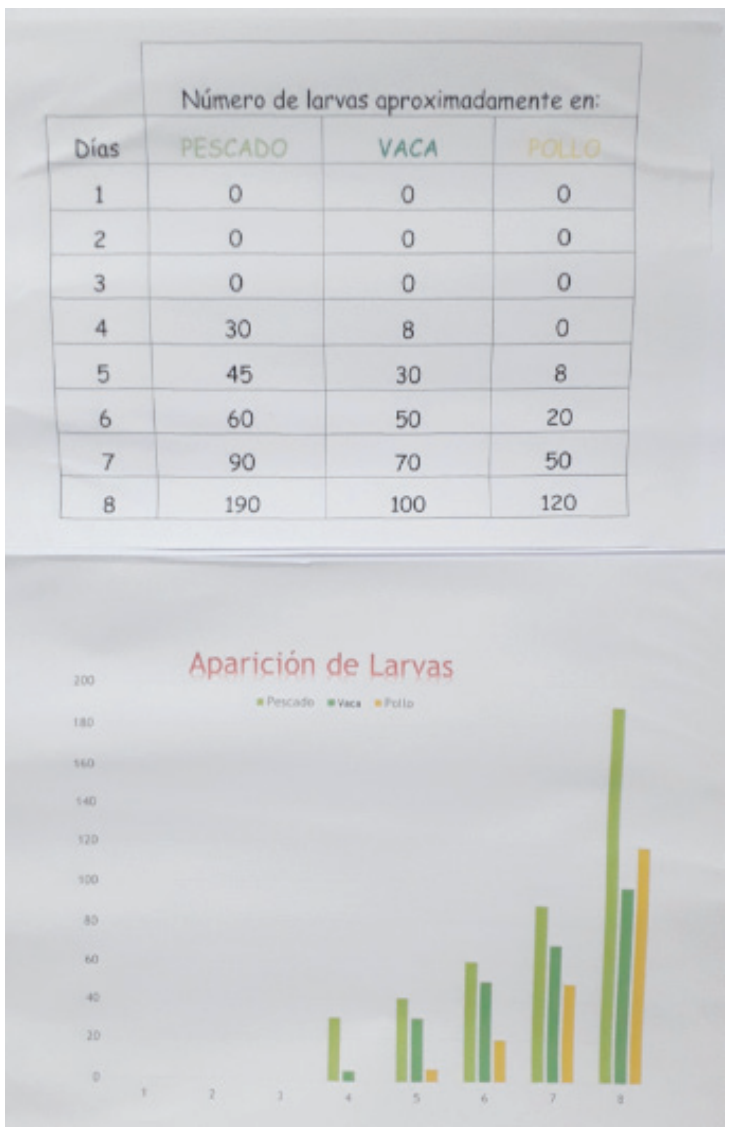


La confección de los mismos fue artesanal, ya que los estudiantes pertenecen a un contexto desfavorable, pero cumplieron en general con criterios establecidos para un póster científico, existiendo diferencias propias, que surgen de los niveles dentro de cada grupo. Un aspecto a mejorar es que en los pósteres de la primera secuencia no diferencian discusión y conclusión, pero no se lo consideró en el puntaje porque no había sido trabajado por la docente en esa secuencia.

Respecto al análisis de los resultados a partir de la tabla elaborada es posible apreciar que los estudiantes realizaron un seguimiento de los cultivos durante ocho días, y efectuaron el recuento de larvas en dicho período, en los tres tipos de carne. Utilizaron un testigo que colocaron en la heladera. Se aprecia un intento por medir la variable tipo de carne y controlar otras variables, aunque no se explicita claramente

En relación a los puntajes obtenidos, en la tabla 5 puede apreciarse los mismos.

Tabla 5. Puntajes obtenidos por los pósteres de 1ero de bachillerato (4to año). Fuente propia.

\begin{tabular}{|c|c|c|c|c|c|c|c|}
\hline Proyecto & Título & Síntesis & Organización & $\begin{array}{l}\text { C. cientí- } \\
\text { fica }\end{array}$ & Originalidad & Sintaxis & Total \\
\hline 1 & $\begin{array}{c}\text { Invasión de } \\
\text { larvas } \\
\end{array}$ & 8 & 10 & 10 & 8 & 10 & 46 \\
\hline 2 & $\begin{array}{c}\text { Hongos en la } \\
\text { fruta }\end{array}$ & 8 & 10 & 10 & 8 & 10 & 46 \\
\hline 3 & $\begin{array}{c}\text { Cromatografía } \\
\text { remolacha }\end{array}$ & 8 & 10 & 10 & 8 & 10 & 46 \\
\hline 4 & $\begin{array}{c}\text { Color en el } \\
\text { morrón }\end{array}$ & 6 & 10 & 9 & 8 & 10 & 43 \\
\hline 5 & $\begin{array}{c}\text { Mitosis en la } \\
\text { cebolla }\end{array}$ & 6 & 10 & 10 & 8 & 10 & 44 \\
\hline 6 & Mitosis & 6 & 8 & 10 & 10 & 10 & 44 \\
\hline 7 & $\begin{array}{c}\text { Células de la } \\
\text { cebolla }\end{array}$ & 7 & 8 & 8 & 8 & 10 & 41 \\
\hline 8 & $\begin{array}{c}\text { Célula animal y } \\
\text { vegetal }\end{array}$ & 6 & 8 & 8 & 7 & 10 & 39 \\
\hline 9 & ADN en saliva & 4 & 8 & 10 & 8 & 6 & 36 \\
\hline 10 & ADN cebolla & 5 & 8 & 10 & 8 & 6 & 37 \\
\hline Total & & 64 & 90 & 95 & 81 & 92 & 422 \\
\hline$\%$ & & 15,2 & 21,3 & 22,5 & 19,2 & 21,8 & 100 \\
\hline
\end{tabular}

La categoría en la que se aprecia mayor dificultad corresponde a la de síntesis de la información y la que obtienen mayor puntaje es la de competencia científica. En esta última categoría se valora que los estudiantes presenten bien formulados el resumen, la pregunta investigable, las hipótesis, los resultados y la conclusión. 
El puntaje logrado por los distintos Proyectos fluctúa entre 36 y 46 puntos, en un total de 50 puntos, sin diferencias significativas. Además de indicar que, todos los estudiantes, han logrado comunicar de forma escrita en un nivel por encima del aceptable sus Proyectos.

Los posters de "invasión de larvas" y "hongos en la fruta" obtienen un total de 46 puntos cada uno, sobre un total de 50 puntos, no encontrándose diferencias entre ambos en las categorías evaluadas.

\subsubsection{Defensa}

Los estudiantes dispusieron de 10 minutos para realizar la defensa de sus proyectos.

La tabla 6 corresponde a los puntajes otorgados por la investigadora, en la última columna se encuentran los promedios de los puntajes adjudicados por las tres docentes y la investigadora.

Tabla 6. Puntajes obtenidos en la defensa de los Proyectos de 1ero de bachillerato. Fuente propia.

\begin{tabular}{|c|c|c|c|c|c|c|}
\hline Proyecto & Título & $\begin{array}{c}\text { Conocimiento } \\
\text { conceptual }\end{array}$ & $\begin{array}{c}\text { Diseño meto- } \\
\text { dológico }\end{array}$ & $\begin{array}{c}\text { Participación } \\
\text { grupo }\end{array}$ & $\begin{array}{c}\text { Puntaje } \\
\text { total }\end{array}$ & $\begin{array}{c}\text { Promedio } \\
\text { tribunal }\end{array}$ \\
\hline 1 & $\begin{array}{l}\text { Invasión de } \\
\text { larvas }\end{array}$ & 9 & 8 & 9 & 26 & 26,3 \\
\hline 2 & $\begin{array}{l}\text { Hongos en la } \\
\text { fruta }\end{array}$ & 9 & 8 & 7 & 24 & 25,6 \\
\hline 3 & $\begin{array}{c}\text { Cromatografía } \\
\text { remolacha }\end{array}$ & 8 & 8 & 8 & 24 & 26 \\
\hline 4 & $\begin{array}{l}\text { Color en el } \\
\text { morrón }\end{array}$ & 8 & 8 & 4 & 20 & 21,3 \\
\hline 5 & $\begin{array}{c}\text { Mitosis en la } \\
\text { cebolla }\end{array}$ & 8 & 10 & 8 & 26 & 26 \\
\hline 6 & Mitosis & 8 & 10 & 10 & 28 & 26 \\
\hline 7 & $\begin{array}{c}\text { Células de la } \\
\text { cebolla }\end{array}$ & 7 & 10 & 8 & 25 & 26,3 \\
\hline 8 & $\begin{array}{c}\text { Célula animal y } \\
\text { vegetal }\end{array}$ & 6 & 8 & 4 & 18 & 22,6 \\
\hline 9 & ADN en saliva & 8 & 10 & 8 & 26 & 26,6 \\
\hline 10 & ADN cebolla & 10 & 10 & 6 & 26 & 26 \\
\hline Total & & 81 & 90 & 72 & 243 & \\
\hline$\%$ & & 33,3 & 37,1 & 29,6 & 100 & \\
\hline
\end{tabular}

La categoría en la que obtienen mejor puntaje es en diseño metodológico y menor puntaje en participación del grupo.

"Invasión de larvas" y "hongos en la fruta" obtuvieron 26.3 y 25,6 puntos respectivamente. La categoría de diseño metodológico es en la que obtienen menor puntaje en relación a los otros proyectos. Lo que puede estar relacionado a que fueron proyectos correspondientes a la primera secuencia. En 
tanto que los proyectos de Mitosis y ADN realizados en la tercera y cuarta secuencia obtienen mejores desempeños en dicha categoría.

Los puntajes totales presentados en la tabla 7, obtenidos por todos los Proyectos oscilan entre 61,6 y 72,3 lo que indica diferencias mínimas entre los estudiantes de ambos grupos.

Tabla 7. Puntajes obtenidos en cada proyecto de 4to año. Fuente propia.

\begin{tabular}{|c|c|c|c|}
\hline Título & Póster & Defensa & Total \\
\hline Invasión de larvas & 46 & 26,3 & 72,3 \\
\hline Hongos en la fruta & 46 & 25,6 & 72 \\
\hline Cromatografía remolacha & 46 & 26 & 64,3 \\
\hline Color en el morrón & 43 & 21,3 & 70 \\
\hline Mitosis en la cebolla & 44 & 26 & 70 \\
\hline Mitosis & 44 & 26,3 & 67,3 \\
\hline Células de la cebolla & 41 & 22,6 & 61,6 \\
\hline Célula animal y vegetal & 39 & 26,6 & 62,6 \\
\hline ADN en saliva & 36 & 26 & 63 \\
\hline ADN cebolla & 37 & & 70 \\
\hline
\end{tabular}

Los puntajes totales varían entre 61,6 y 71,6, en un total de 90 puntos.

En los resultados totales de los posters y defensas, en la tabla 7 se percibe que invasión de larvas fue el proyecto que obtuvo mejor puntaje.

Puede interpretarse que existió una involución de los trabajos, comenzando el año con trabajos muy buenos para luego ir declinando en el nivel de los mismos. Lo cual invita a "cristalizar" con otros datos y profundizar en el análisis. La docente selecciona para la Muestra proyectos de diferentes estudiantes, para que todos los estudiantes puedan participar, algunos estudiantes presentan proyectos de la primera secuencia y otros estudiantes de las siguientes. Es necesario tomar datos provenientes de los resultados obtenidos en la propuesta diagnóstica por los estudiantes del proyecto "invasión de larvas". Los resultados individuales se observan en la tabla 8 .

La numeración proviene de la rúbrica, en la cual el tres representa el excelente, 2 muy bueno, 1 aceptable, y 0 corresponde al insuficiente o "debe mejorar".

Los tres estudiantes obtienen puntajes suficientes tanto en el ítem 1 como en el ítem 4 . Incluso en el ítem 4 , los puntajes de dos de ellos corresponden al excelente y el tercero muy bueno. 
En la escala que se utiliza para llevar el puntaje de la rúbrica a calificaciones, 1 a 12 es insuficiente, de 13 a 17 es aceptable, de 18 a 22 muy bueno y de 23 a 27 excelentes.

Tabla.8. Resultados individuales en la propuesta diagnóstica de los estudiantes que elaboraron el proyecto invasión de larvas. Fuente propia.

\begin{tabular}{|c|c|c|c|c|c|c|c|c|c|c|}
\hline $\begin{array}{l}\text { Estu- } \\
\text { diante }\end{array}$ & $\begin{array}{c}\text { 1.Iden- } \\
\text { tificar } \\
\text { cuestio- } \\
\text { nes cien- } \\
\text { tíficas. } \\
\text { Explicar } \\
\text { fenó- } \\
\text { menos } \\
\text { científi- } \\
\text { camente. } \\
\text { Utilizar } \\
\text { eviden- } \\
\text { cia cien- } \\
\text { tífica. }\end{array}$ & $\begin{array}{c}\text { 2.Iden- } \\
\text { tificar } \\
\text { pro- } \\
\text { blemas } \\
\text { cientí- } \\
\text { ficos. } \\
\text { Definir } \\
\text { objeti- } \\
\text { vos de } \\
\text { investi- } \\
\text { gación. } \\
\text { For- } \\
\text { mular } \\
\text { hipóte- } \\
\text { sis. }\end{array}$ & $\begin{array}{l}\text { 3.Bus- } \\
\text { car } \\
\text { infor- } \\
\text { ma- } \\
\text { ción } \\
\text { dife- } \\
\text { rentes } \\
\text { fuen- } \\
\text { tes y } \\
\text { valo- } \\
\text { rarla } \\
\text { de } \\
\text { forma } \\
\text { crítica } \\
\text { y re- } \\
\text { flexi- } \\
\text { va. }\end{array}$ & $\begin{array}{c}\text { 4.Iden- } \\
\text { tificar } \\
\text { varia- } \\
\text { bles. } \\
\text { Dise- } \\
\text { ñar una } \\
\text { me- } \\
\text { todo- } \\
\text { logía. } \\
\text { Rea- } \\
\text { lizar } \\
\text { expe- } \\
\text { rien- } \\
\text { cias }\end{array}$ & $\begin{array}{l}\text { 5.Pro- } \\
\text { cesar } \\
\text { los } \\
\text { resul- } \\
\text { tados } \\
\text { en } \\
\text { dis- } \\
\text { tintos } \\
\text { for- } \\
\text { matos } \\
\text { (ta- } \\
\text { blas, } \\
\text { gráfi- } \\
\text { cos) }\end{array}$ & $\begin{array}{l}\text { 6.For- } \\
\text { mular } \\
\text { con- } \\
\text { clu- } \\
\text { siones }\end{array}$ & $\begin{array}{l}\text { 7.Dar } \\
\text { a co- } \\
\text { nocer } \\
\text { los } \\
\text { resul- } \\
\text { tados }\end{array}$ & $\begin{array}{c}\text { 8.Tra- } \\
\text { bajar en } \\
\text { equipo, } \\
\text { respetar } \\
\text { y valorar } \\
\text { las ideas } \\
\text { de los } \\
\text { compa- } \\
\text { ñeros y } \\
\text { tomar } \\
\text { decisio- } \\
\text { nes. }\end{array}$ & $\begin{array}{l}\text { 9.Identificar } \\
\text { prácticas } \\
\text { científicas } \\
\text { beneficiosas } \\
\text { para la ma- } \\
\text { yoría de los } \\
\text { ciudadanos. }\end{array}$ & Puntaje \\
\hline 4.DF & 3 & 0 & 3 & 2 & 2 & 1 & 1 & 3 & 2 & 17 \\
\hline $15 \mathrm{NS}$ & 1 & 1 & 2 & 3 & 1 & 1 & 1 & 3 & 1 & 14 \\
\hline $18 \mathrm{PF}$ & 1 & 1 & 2 & 3 & 1 & 1 & 1 & 3 & 1 & 14 \\
\hline
\end{tabular}

De acuerdo a la escala utilizada para calificar, los tres estudiantes obtienen calificación aceptable. Se ubican dentro de los ocho estudiantes de los veintitrés, del grupo 2, que obtienen calificaciones aceptables. De estos datos es posible interpretar que la docente seleccionó de la primera secuencia, los posters de los mejores estudiantes, ya que fueron los mejores trabajos al inicio del año y selecciona posters de los demás estudiantes para las siguientes secuencias, para que todos los estudiantes puedan presentar sus trabajos y que estén representadas todas las secuencias. Los estudiantes que no logran al inicio resultados favorables, si alcanzan dicho nivel en el transcurso del año.

\section{Discusión}

La investigación acción implica un trabajo en equipo con la docente en la planificación de la secuencia, se logra consenso e importantes aportes de la misma. Se crea una comunidad de aprendizaje, con las tres docentes, desde un enfoque socio crítico y se alcanza la autonomía en el transcurso del año en la propuesta de actividades dentro del modelo de aprendizaje por indagación, así como un pensa- 
miento crítico, que se explicita en acción y reflexión, como lo expresan Carr y Kemmis (1988, p. 62) No obstante ello, en el presente artículo solamente se analiza el trabajo de una de las docentes.

Los resultados obtenidos responden a la pregunta planteada por Domènech-Casal (2017, p. 55) “¿es posible desarrollar una programación de toda una materia a partir de metodologías de trabajo por proyectos de indagación?", su investigación evidencia que es posible. En su caso se implementaron ocho proyectos en el curso estudiado. También en esta investigación se planificaron los tres cursos mediante proyectos: cinco en primer año, seis en tercer año y seis en cuarto año, alcanzándose un cambio muy importante en la planificación de las secuencias didácticas.

Todas las secuencias elaboradas son contextualizadas y se plantean situaciones problema que parten de la realidad de los estudiantes. En cada una de ellas los estudiantes elaboran preguntas investigables de acuerdo a sus intereses. Harlen (2011) enuncia que, si se desea que los estudiantes desarrollen actitudes positivas hacia el aprendizaje de las ciencias, es necesario que sus indagaciones estén vinculadas con la realidad.

Respecto a la progresión en el desarrollo de la competencia científica, se observan avances, no obstante en la dimensión dos, en la cual se encuentra la capacidad de formular preguntas investigables, Sanmartí y Márquez Bargalló (2012) indican que formular buenas preguntas de investigación es la característica más importante de un científico, pero el aprendizaje de la formulación de las mismas resulta una tarea difícil según los resultados de la investigación en educación, por lo que se enfatiza la importancia de los resultados obtenidos en las preguntas elaboradas

Puede apreciarse que algunas dimensiones se desarrollan mejor que otras. Yus Ramos, Fernández Navas, Gallardo Gil, Barquín Ruiz, Sepúlveda y Serván Núñez (2013) destacan que, si se acepta la definición holística de competencia, no puede pensarse que por dominar una capacidad el aprendiz ya es competente, se necesitan dominar todas las capacidades para poder resolver problemas reales, por lo cual, si bien se observan avances en algunas capacidades y dimensiones, aún no se logra el desarrollo de la competencia científica desde una concepción holística de la misma.

Uno de los aspectos que se pretende investigar es el vinculado con las características del modelo didáctico de aprendizaje por indagación en relación a la atención a la diversidad e inclusividad. De acuerdo a lo expresado por Barrio (2008), atender a la diversidad implica planear un currículo y metodologías que se adapten a dicha diversidad escolar. Luego de aplicado el trabajo con proyectos, al finalizar el curso los grupos mejoran, y se igualan los resultados, las diferencias iniciales entre los grupos del mismo grado se desvanecen por lo cual se puede apreciar que el trabajo con proyectos de indagación coadyuva a la inclusividad en el contexto que se desarrolla. Se observan mejoras en la evolución de diferentes capacidades de la competencia científica en todos los estudiantes que participaron en la Muestra. 


\section{Conclusiones}

\subsection{Desarrollar secuencias de aprendizajes duales a través del diseño de proyectos dentro del modelo didáctico de aprendizaje por investigación.}

Se desarrollan secuencias duales que involucran aspectos didácticos y epistemológicos. En la secuencia descripta se puede apreciar que a partir de los contenidos curriculares y teniendo en cuenta el interés de los estudiantes, fue posible contextualizar, investigar y lograr la motivación de los mismos.

Los Proyectos se desarrollan en el aula y algunas actividades las cumplen como tareas domiciliarias, lo que permite afirmar que es posible abordar el currículo a través de varios proyectos en éstos grupos.

\subsection{Describir el avance que logran realizar los estudiantes de los grupos selecciona- dos, en las distintas dimensiones y capacidades que incluye la competencia científica.}

De las siete preguntas elaboradas en la primera clase observada, solamente dos de ellas son investigables, las demás necesitan que se reformulen. Estos resultados muestran la dificultad en esta dimensión de la competencia científica.

En la jornada de presentación y defensa de los Proyectos todos los estudiantes demuestran haber logrado un avance en las diferentes dimensiones y el aprendizaje en los temas tratados.

En la evaluación del póster, la categoría en la que logran mejor puntaje corresponde a competencia científica. Asimismo, en la defensa obtienen mejor puntaje en la categoría diseño metodológico.

Los puntajes finales logrados oscilan entre 61,6 y 71,6 en un total de 90 puntos lo que indica que en el transcurso del año realizaron una evolución en el desarrollo de la competencia, sin alcanzar aún la excelencia en la elaboración y comunicación de los Proyectos.

Es de destacar que a través de la evaluación de los posters y de las defensas de los Proyectos es posible apreciar que se desvanecen las diferencias iniciales detectadas por la propuesta diagnóstica en las diferentes capacidades y dimensiones de la competencia científica para los estudiantes de los grupos seleccionados, ya que los puntajes obtenidos no indican diferencias importantes en los resultados.

Se puede interpretar que el trabajo con Proyectos de Introducción a la Investigación en estos grupos actúa de forma inclusiva ya que logra neutralizar las desigualdades iniciales en la competencia científica entre los estudiantes, igualando hacia mejores resultados. La interpretación se representa a través de la figura 4. 

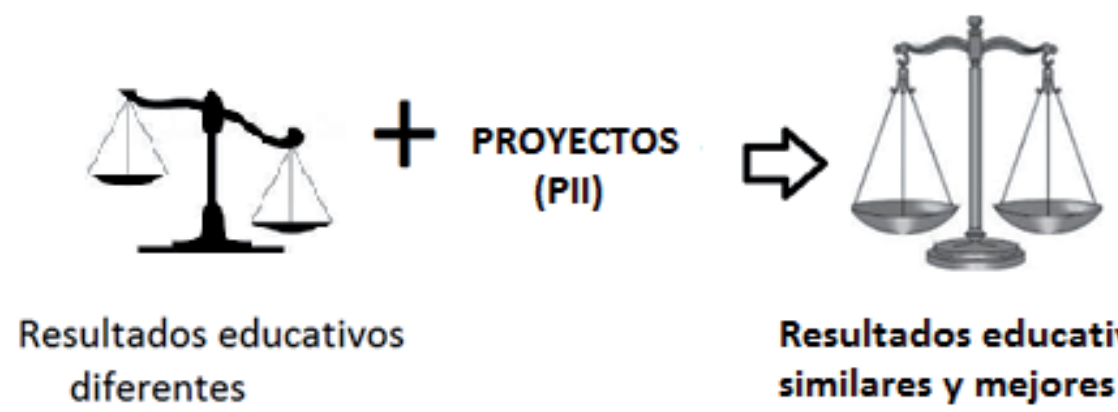

Resultados educativos similares y mejores

Figura 4. Competencia científica en los estudiantes antes y luego del trabajo con Proyectos de Introducción a la Investigación. Fuente propia.

Los resultados obtenidos ameritan que se continúe investigando sobre este modelo didáctico, dado la evidencia relevada sobre su vinculación en la mejora de la competencia científica.

Una evaluación final individual puede arrojar mayor claridad sobre el avance que logran realizar cada uno de los estudiantes y permitirá confrontar de mejor forma la interpretación de éstos resultados.

\section{Referencias}

Alonso Tapia, J. (2005) Motivación para el aprendizaje: la perspectiva de los alumnos. En Ministerio Educación y Ciencia. La orientación escolar en centros educativos. (pp. 209-242). Madrid: MEC. Disponible en: http://www.orientacioncadiz.com/files/EOEs/EOE\%20 $\underline{\text { ARCOS/2005 motivacion para el aprendizaje Perspectiva_alumnos.pdf }}$

Arango, N., Chaves, M., Feinsinger, P. (2009) Principios y práctica de la Enseñanza de Ecología en el Patio de la Escuela. Instituto de Ecología y Biodiversidad. Fundación Senda Darwin, Santiago, Chile. 136 pp. Disponible en: http://nazari.devmg.com/wp-content/ uploads/2015/05/Manual-EEPE.pdf

Barrio, J. L. (2008). Hacia una Educación Inclusiva para todos. Revista Complutense de Educación, 20 (1), 13-31.

Caamaño, A., Corominas, J. (2004) ¿Cómo abordar con los estudiantes la planificación y realización de trabajos prácticos investigativos? Alambique, 39, 52-63. Disponible en:https:// www.researchgate.net/publication/39207519 Como abordar con los estudiantes la planificacion de los trabajos practicos investigativos 
Caamaño, A. (2012) La investigación escolar es la actividad que mejor integra el aprendizaje de los diferentes procedimientos científicos. En E. Pedrinaci (coord.) Once ideas claves. El desarrollo de la competencia científica. (pp. 127-143). Barcelona: Graó.

Cañal, P. (2011) Biología y Geología. Investigación, innovación y buenas prácticas. Barcelona: Ed. Graó.

Carr, W., Kemmis, S. (1988) Teoría crítica de la enseñanza. Barcelona: Martínez Roca.

Chin, C., Osborne, J. (2008). Students' questions: a potential resource for teaching and learning science. Studies in Science Education, 44(1), 1-39. Disponible en: https://www.tandfonline.com/doi/abs/10.1080/03057260701828101

Couso, D. (2011) Las secuencias didácticas en la enseñanza y el aprendizaje de las ciencias: modelos para su diseño y validación. En A. Caamaño (coord.) Didáctica de la física y la química. (pp. 57-74) Barcelona: Graó

Couso, D. (2013). La elaboración de unidades didácticas competenciales. Alambique, Didáctica de las Ciencias Experimentales, 74, 12-24. Disponible en:http://cmap.unavarra.es/ $\underline{\text { rid=1RKTWRZXR-1Z8TJRV-2V6/DIGNACOUSO ELABRACION UD.pdf }}$

Domènech-Casal, J. (2017). Proyectando BioGeo. Un itinerario en indagación y naturaleza de la ciencia. Alambique Didáctica de las Ciencias Experimentales, 89, 54-61. Disponible en:https://www.researchgate.net/publication/318041571 Proyectando BioGeo un itinerario de trabajo por proyectos contextualizados basado en la indagacion y la Naturaleza de la Ciencia

Harlen, W (2011). Aprendizajes y enseñanza de ciencias basados en la indagación. Inglaterra: Universidad de Bristol. Disponible en:http://www.ecbichile.cl/home/wp-content/ uploads/2012/05/Aprendizaje-y-ensen\%23U0303anza-de-ciencias-basados-en-laindagacio\%23U0301n..pdf

Franco-Mariscal, A.J., Blanco-López, A., España-Ramos, E. (2014) El desarrollo de la competencia científica en una unidad didáctica sobre la salud bucodental. Diseño y análisis de tareas. Enseñanza de las Ciencias, 32 (3), 649-667. Disponible en:https://core.ac.uk/ download/pdf/38989802.pdf

Franco-Mariscal, A. J. (2015) "Competencias científicas en la enseñanza y el aprendizaje por investigación. Un estudio de caso sobre corrosión de metales en secundaria”. Enseñanza 
de las Ciencias, 33 (2), 231-252. Disponible en:https://www.raco.cat/index.php/Ensenanza/article/viewFile/293274/381774

Pedrinaci, E. (comp.) (2012) Once ideas claves. El desarrollo de la competencia científica. Barcelona: Graó.

Quintanilla, M.(comp) (2014) Las Competencias de Pensamiento Científico desde las "emociones, sonidos y voces" del aula. Santiago de Chile: Bellaterra

Richardson, L. (1997). Fields of play: Constructing an academic life. New Brunswick NJ: Rutgers University Press.

Sánchez, M. (2013) Aprendizaje colaborativo basado en proyectos en ingeniería. Revista Iberoamericana para la Investigación y el Desarrollo Educativo,10. Disponible en:https://www. researchgate.net/profile/Mario Roberto Gatica/publication/280886520 Las Competencias de pensamiento cientifico desde las emociones sonidos y voces del aula/ links/55ca9b1a08aea2d9bdcc0586/Las-Competencias-de-pensamiento-cientifico-desde-las-emociones-sonidos-y-voces-del-aula.pdf

Sanmartí, N. y Márquez Bargalló, C. (2012). Enseñar a plantear preguntas investigables. Alambique Didáctica de las Ciencias Experimentales, 70, 27-36. Disponible en:http://gent.uab. cat/conxitamarquez/sites/gent.uab.cat.conxitamarquez/files/Ense\%C3\%B1ar\%20a\%20 plantear\%20preguntas\%20investigables.pdf

Yus Ramos, R., Fernández Navas, M., Gallardo Gil, M., Barquín Ruiz, J., Sepúlveda, M. P. y Serván Núñez, M. J. (2013). La competencia científica y su evaluación. Análisis de las pruebas estandarizadas de PISA. Revista de Educación, 360, 557-576. http://www.revistaeducacion.mec.es/doi/360 127.pdf

Travé, Pozuelo, Cañal, (2007) ¿Cómo enseñar investigando? Análisis de las percepciones de tres equipos docentes con diferentes grados de desarrollo profesional. Revista Iberoamericana de Educación, 1, 1-24. Disponible en: https://idus.us.es/xmlui/handle/11441/16326

Tobón, S., Pimienta, J., García Fraile, J. (2010) Secuencias didácticas: aprendizaje y evaluación de competencias. México: Pearson Educación.

Zabala, A., Arnau, L. (2014) Métodos para la enseñanza de las competencias. Barcelona: Ed. Graó. 